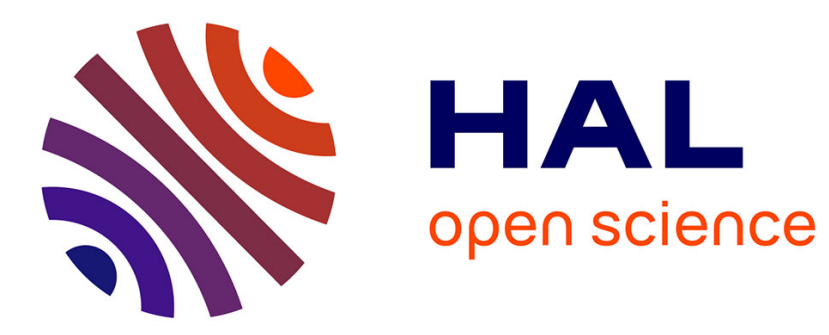

\title{
Magnetoelastic modeling of core-shell spin-crossover nanocomposites
}

\author{
Hassane Oubouchou, Yogendra Singh, Kamel Boukheddaden
}

\section{To cite this version:}

Hassane Oubouchou, Yogendra Singh, Kamel Boukheddaden. Magnetoelastic modeling of core-shell spin-crossover nanocomposites. Physical Review B, 2018, 98 (1), 10.1103/PhysRevB.98.014106 . hal03044102

\section{HAL Id: hal-03044102 \\ https://hal.science/hal-03044102}

Submitted on 25 Jun 2021

HAL is a multi-disciplinary open access archive for the deposit and dissemination of scientific research documents, whether they are published or not. The documents may come from teaching and research institutions in France or abroad, or from public or private research centers.
L'archive ouverte pluridisciplinaire HAL, est destinée au dépôt et à la diffusion de documents scientifiques de niveau recherche, publiés ou non, émanant des établissements d'enseignement et de recherche français ou étrangers, des laboratoires publics ou privés. 


\title{
Magnetoelastic modeling of core-shell spin-crossover nanocomposites
}

\author{
Hassane Oubouchou, ${ }^{1,2}$ Yogendra Singh, ${ }^{1}$ and Kamel Boukheddaden ${ }^{1, *}$ \\ ${ }^{1}$ Groupe d'Etudes de la Matière Condensée, Université de Versailles, Université Paris-Saclay, CNRS UMR 8635, \\ 45 Avenue des Etats Unis 78035 Versailles, France \\ ${ }^{2}$ Ecole Polytechnique d'Architecture \& d'Urbanisme Route de Beaulieu, BP 177, 16010 El Harrach, Algeria
}

(Received 2 February 2018; revised manuscript received 6 June 2018; published 11 July 2018)

\begin{abstract}
Spin-crossover (SCO) solids have been studied from magnetic and structural viewpoints under the form of powder, single crystals, and nanoparticles. Recently, an important progress made in chemistry allowed the design of spin-crossover nanocomposites, combining the properties of two types of spin-crossover solids having different properties, for example, different transition temperatures, ligand fields, etc. In this work, we sketch theoretical investigations based on an elastic description of a SCO nanocomposite, aiming to reproduce the experimental results of literature which exhibited two-step and even three-step spin transitions. Our results not only reproduce the experimental features but also allow a better understanding of the interplay between the elastic and electronic structures of the two components of the SCO nanocomposite. The obtained data offer serious possibilities of extensions to several configurations of nanocomposites according to the shape of the lattice and the relative sizes of the two components under study.
\end{abstract}

DOI: 10.1103/PhysRevB.98.014106

\section{INTRODUCTION}

The thermally induced spin-crossover (SCO) transition between the low spin (LS) and the high spin (HS) states of $\mathrm{Fe}(\mathrm{II})$ complexes with suitable ligands are examples of molecular bistable solids. They have been studied [1-9] for many years for their promising applications as sensors of pressure [10], displays [11], memories, and molecular switches [12]. During the last decade, compounds with multistep spin conversion remained quite rare and have attracted increasing attention in theoretical and experimental studies [13-17], due to their possibility to build up three-byte electronics.

Multistep SCO behavior results from structural ordering or/and the existence of a multistability in the molecule itself, like in binuclear SCO systems $[18,19]$. In contrast, some multistep SCO systems consist of an asymmetric unit containing two or more nonequivalent sites [20,21], having different local environments. At the macroscopic scale, the competition or the interplay between the two types of SCO sites manifest through the existence of an intermediate phase associated with partial conversion from HS to LS states. It is worth noticing that the processing of SCO materials with two inequivalent sites, although it is possible, it is hardly controllable and most of the time the behavior of the high-spin fraction is hardly predictable. That is why recently, chemists and physicists started designing well controllable and reproducible SCO core-shell nanocomposites, made of two SCO materials, thus combining the SCO properties of both the core and the shell components in the same system $[22,23]$. Till now, the interest in the finite size effects properties [24-27] and in the manipulation [28], the design, and visualization [29] of small objects at the nanoscale is rapidly growing since the adapted near-field technologies

*kamel.boukheddaden@uvsq.fr are now available. This was the case, for example, of prussian blue analogs for which core-shell nanoparticles have been synthesized, and their thermal and photostriction properties have been investigated as a function of the thickness and the nature of the core and the shell [30-34].

With the development of nanotechnologies and the availability of experimental results, several types of nanocomposites integrating the SCO properties have been designed by embedding SCO nanoparticles within organic polymers [35-39] in biopolymers [40] or in mesoporous silica matrices [41-44]. Core-shell nanocomposites have attracted considerable attention also from the theoretical point of view, where several models have been proposed to investigate the size effect on the SCO properties of nanoswitchable materials, among which the well-known Ising-like model [45] which has been used in this context by Kawamoto et al. [46,47]. Later on we extended this model to account for specific boundary conditions $[48,49]$ in square-shaped SCO nanoparticles that we solved by MC simulations. Other theoretical developments, based on the Ising-like model, investigating the distribution of HS and LS domains in the thermal hysteresis region of SCO nanoparticles using FORC diagrams were also reported [50].

Furthermore, taking a benefit from the development of spinphonon models [51-54] and the extension of these models accounting for the volume change upon spin transition designed in several groups [55-57], we elaborated the electroelastic model [58], which takes into account the coupling between the spin state change of the molecule (from LS to HS) and the change of the local molecular volume in the course of the spin transition and other models including the role of surface relaxations [59,60] and the particle environment [61-63],

In 2013 we adapted the electroelastic model to the case of SCO nanoparticles [58], in which we have considered that the SCO atoms at the surface are kept in the HS state, due to their specific environment. In this paper we justified this hypothesis 
by the fact that the SCO molecules at the surface might be coordinated to water molecules, oxygen atoms, or pollution leading to decrease their ligand field, which in turn stabilizes the HS state. However, we pointed out in the conclusion that several other effects may also lead to a specific behavior of the surface, among which surface phonons which are different from those of the bulk, as well as structural properties, including the possible existence of surface reconstructions. As a result of our simple assumption, the thermal hysteresis moves according to the atomic surface/volume ratio, in very good agreement with the available experimental data of Volatron et al. [64]. Since this pioneering work, the electroelastic model has been extended to incorporate the case of core-shell SCO nanoparticles, made of an active SCO core and an inert shell, where we investigated the detailed properties of the SCO core as a function of the elastic properties (rigidity, lattice parameter misfit, and size) of the shell [65-70]. It is worth mentioning that other groups investigated the problem of active core-shell SCO nanoparticles by considering hollow core-shell configurations, or by studying their vibrational properties as well the role of the elastic interface energy in the stability of the nanoparticles [71-73]. Other developments which included the role of surface relaxations $[60,74]$ and the particle environment $[62,65,75]$ have also emerged in recent years.

It is important to mention that back in 2013 we had pointed out the possibility of several other reasons leading to renormalizing the ligand field at the surface of nanoparticles or to maintaining the spin states of SCO atoms at the surface. To our best knowledge, there were no experimental proofs leading to discriminate between these possible causes. Indeed, most of the experimental studies on SCO nanoparticles, if not all, have been realized on a large number of nanoparticles. Thus, for example, when Mossbauer spectroscopy studies (where around $20 \mathrm{mg}$ of sample are necessary) are performed on SCO nanoparticles (average density $\sim 2 \mathrm{~g} / \mathrm{cm}^{3}$ ) it is easy to evaluate their number at a minimum is around $\sim 10^{15}$, which then lead to obtaining an averaged response over nanoparticles with different size, shapes, surface structures, etc. In short, as long as experimental studies on one single nanoparticle, like in magnetic systems [76,77], are not realized, this issue will not be definitely resolved.

In this theoretical contribution, we analyze the case of spin-crossover nanocomposites (both core and shell become active), which became very popular in recent years. Experimental investigations in this field have been performed by several groups [14,23,78-81], among whom, Wang et al. [14], in particular, have succeeded to grow high-quality spincrossover nanocomposites by mixing two kinds of SCO materials, namely $\left[\mathrm{Fe}\left(\mathrm{NH}_{2} \text {-trz }\right)_{3}\right]\left(\mathrm{BF}_{4}\right)_{2}$ and $\left[\mathrm{Fe}(\mathrm{Htrz})_{2}(\mathrm{trz})\right]\left(\mathrm{BF}_{4}\right)$, which have different SCO properties, since the former has a transition temperature $T_{1}=260 \mathrm{~K}$ and a hysteresis width of $4 \mathrm{~K}$, while the latter shows a transition temperature $T_{2}=368 \mathrm{~K}$ and an associated thermal hysteresis width of $46 \mathrm{~K}$. Thus, these two materials have different ligand fields and most likely different strength of cooperativity between the molecules. In the experiment, the core is constituted by the material which has the smaller ligand field, which was then covered by the second material (stronger ligand field) in rather heteroepitaxial conditions. Although the strong one-dimensional (1D) character of the polymeric materials leads to the formation of nanorod blocks of first material, covered by cylindrical-shaped second material; the ensemble forms a coaxial cablelike configuration. The magnetic studies of the formed nanocomposite, reported by the authors in Refs. [14,79], showed the occurrence of clearly two transition temperatures on heating, located at 288 and $314 \mathrm{~K}$. Thus, the transition temperature of the first constituent (the core) increased by $28 \mathrm{~K}$ while that of the second material (the shell) decreased by $54 \mathrm{~K}$, due to the elastic interplay between the core and the shell nanostructures. Furthermore, a very gradual and third transition involving a small fraction of molecules occurs at $347 \mathrm{~K}$; the latter was explained as due to the occurrence of a mixture material.

In this contribution we propose to describe an ideal situation of active core-shell SCO nanoparticle, made of two SCO constituents able to convert between LS and HS states, using the electroelastic model, with electronic and elastic parameters adapted to emulate as far as possible realistic data of SCO nanocomposites. Thus, we have chosen the core to have a smaller transition temperature than that of the shell, as core and shell constitute two different SCO compounds with specific ligand fields and elastic constants. The study of the core-shell nanoparticles is realized by Monte Carlo simulations in twodimensional (2D) lattices with square symmetry, with the aim to reveal the role of the elastic interaction between the two constituents on their transition temperatures on the one hand, and the effect of their interplay on the mode of the nucleation and growth of their spin states during their thermal switching on the other hand.

The paper is organized as follows: in Sec. II we present the model, describe the simulation technique, and explain our choice of interaction parameter values. Section III is devoted to the presentation of the results of the simulations and to their discussion, and in Sec. IV we conclude and outline some possible developments of the present work.

\section{THE MODEL}

To simulate a SCO nanocomposite, we considered a square lattice core-shell nanoparticle, where either in the core or the shell the atoms can switch between the LS and the HS states. The core has a square shape containing $N_{\mathrm{C}} \times N_{\mathrm{C}}$ sites. The shell has the shape of a thick ring surrounding all the core [see Fig. 1(a)] and the number of atoms along its width is denoted $N_{\mathrm{S}}$. As a result, the number of shell atoms is easily derived as $\left(N_{\mathrm{C}}+2 N_{\mathrm{S}}\right)^{2}-N_{\mathrm{C}}^{2}=4 N_{\mathrm{S}}\left(N_{\mathrm{C}}+N_{\mathrm{S}}\right)$, and the total number of atoms of the composite is $N^{2}$, where $N=N_{\mathrm{C}}+2 N_{\mathrm{S}}$.

We consider here a square lattice [Fig. 1(a)] of SC units in which each of them may have two states, HS and LS, described by an associated two-states fictitious spin $S$, with respective values $S_{i}=+1$ and $S_{i}=-1$. The present model includes the change of the local volume of the SCO molecules during their switching from one state to another, through the coupling of their electronic and elastic properties. Each atom located at site " $i$ " is described by its spin state $S_{i}= \pm 1$ and its coordinates $\left(x_{i}, y_{i}\right)$. The atoms are constrained to move only in the plane as a result of their spin state changes, which affects their equilibrium positions. The reader can find a review of the original electroelastic model for which we have already discussed in detail some of its thermodynamic properties in recent works [65,82]. 

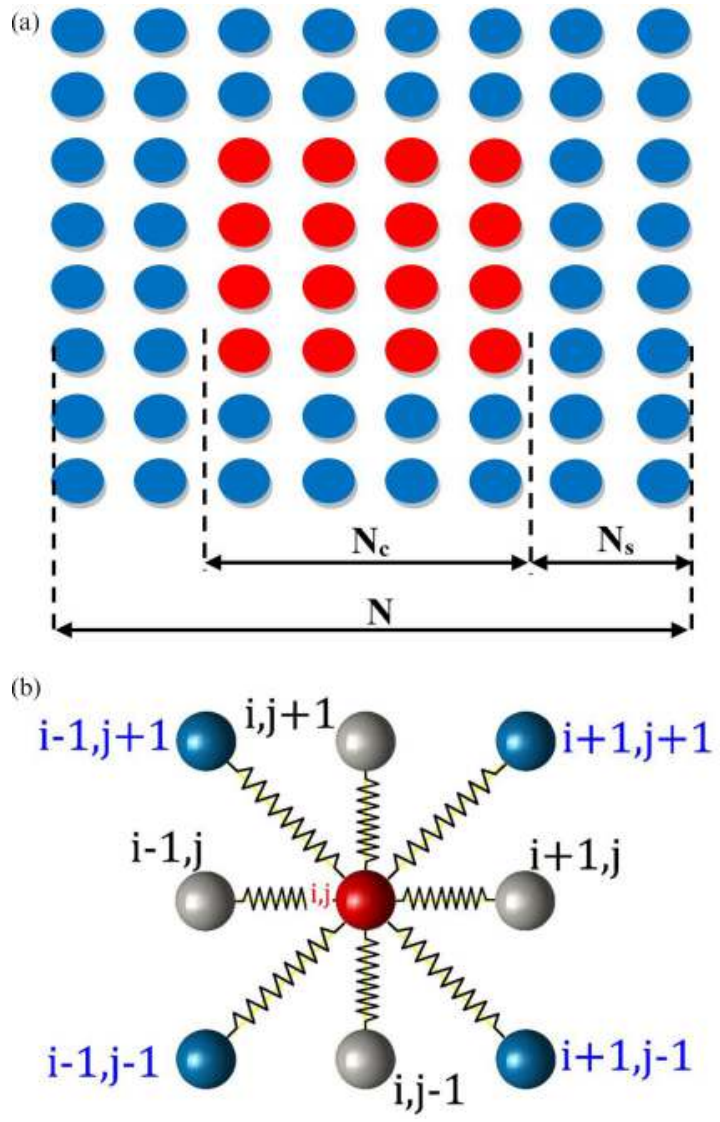

FIG. 1. (a) Schematic structure of the SCO nanocomposite. Blue and red dots are two spin-crossover sites belonging to different materials. (b) The configuration of the elastic interaction in the two dimensions square model considered in this study. The different colors represent, the central, nn and nnn atoms.

Here the model is a bit more general since we extend it to account for the thermodynamic features of SCO nanocomposites, which consists in the coupling of two SCO materials in a particular lattice configuration. The general Hamiltonian [67] describing SCO lattices is written as follows:

$$
H=H_{\text {core }}+H_{\text {shell }}+H_{\text {inter }},
$$

where the core, shell, and interface contributions is written as follows:

$$
\begin{aligned}
H_{\text {core }}= & \sum_{i} \frac{1}{2}\left[\Delta_{\mathrm{C}}-k_{B} T \ln g\right] S_{i}+A_{\mathrm{C}} \sum_{(i, j)}^{\mathrm{nn}}\left[r_{i j}-R_{0}\left(S_{i}, S_{j}\right)\right]^{2} \\
& +B_{\mathrm{C}} \sum_{(i, k)}^{\mathrm{nnn}}\left[r_{i k}-R_{0}^{\prime}\left(S_{i}, S_{k}\right)\right]^{2}, \\
H_{\text {shell }}= & \sum_{i} \frac{1}{2}\left[\Delta_{\mathrm{S}}-k_{B} T \ln g\right] S_{i}+A_{\mathrm{S}} \sum_{(i, j)}^{\mathrm{nn}}\left[r_{i j}-R_{0}\left(S_{i}, S_{j}\right)\right]^{2} \\
& +B_{\mathrm{S}} \sum_{(i, k)}^{\mathrm{nnn}}\left[r_{i k}-R_{0}^{\prime}\left(S_{i}, S_{k}\right)\right]^{2}, \\
H_{\text {inter }}= & A_{i} \sum_{(i, j)}^{\mathrm{nn}}\left[r_{i j}-R_{0}\left(S_{i}, S_{j}\right)\right]^{2}+B_{i} \sum_{(i, k)}^{\mathrm{nnn}}\left[r_{i k}-R_{0}^{\prime}\left(S_{i}, S_{k}\right)\right]^{2} .
\end{aligned}
$$

In the shell and core Hamiltonians, the first term expresses the effective ligand field energy which contains the contribution of the ligand field $\Delta\left(\Delta_{\mathrm{S}}\right.$ for the shell and $\Delta_{\mathrm{C}}$ for the core) and that of the entropy of the HS state $k_{B} T \ln \mathrm{g}$, where $g$ is the degeneracy ratio between the HS and the LS states assumed here, the same for the core and shell constituents. This degeneracy originates from the change of spin states and intra- and intermolecular vibrations spectra during the LS to HS conversion. In the present model, $A_{\mathrm{C}}, B_{C}\left(A_{\mathrm{S}}, B_{\mathrm{S}}\right)$ represent the elastic constants of the core (the shell) between the first nearest neighbors (nn) and the next nearest neighbors (nnn), respectively. Similarly, the elastic constants in the interface region are noted $A_{i}$ and $B_{i}$. For the sake of simplicity, all elastic constants are taken as constant over the lattice sites. In addition, to reduce the number of free parameters in the model, we have chosen $A_{\mathrm{C}}=A_{\mathrm{S}}=A_{i}$ and $B_{\mathrm{C}}=B_{\mathrm{S}}=B_{i}$.

An important aspect of the present model is the change in the lattice parameter along the spin transition between LS and HS. For that, we write the equilibrium distances between two neighboring sites as depending on the spin states, as we have previously introduced in [58]. Let us take two nodes of the lattice, located at sites $(i, j)$ and $\left(i^{\prime}, j^{\prime}\right)$, which can be nn or nnn. They are connected by springs and the instantaneous distance between them is written $r\left(i, j \mid i^{\prime}, j^{\prime}\right)=\left|\vec{r}\left(i^{\prime}, j^{\prime}\right)-\vec{r}(i, j)\right|$, whose indexes are $\left(i^{\prime}=i, j^{\prime}=j \pm 1\right.$ and $i^{\prime}=i \pm 1$, $\left.j^{\prime}=j\right)$ for the $\mathrm{nn}$ or $\left(i^{\prime}=i \pm 1, j^{\prime}=j \pm 1\right)$ for the nnn. The molecules (here, the sites) interact via elastic springs, as depicted in Fig. 1(b), and the equilibrium bond lengths of which, denoted $R_{0}\left(S_{i}, S_{j}\right)\left[R_{0}^{\prime}\left(S_{i}, S_{k}\right)\right]$ between two nn (nnn) spin-crossover atoms located at sites $i$ and $j(i$ and $k$ ) and having the spin state $S_{i}$ and $S_{j}\left(S_{i}\right.$ and $\left.S_{k}\right)$ are given by

$$
\begin{aligned}
& R_{0}\left(S_{i}, S_{j}\right)=R_{0}^{\mathrm{HL}}+\frac{\delta_{R}}{4}\left(S_{i}+S_{j}\right), \\
& R_{0}^{\prime}\left(S_{i}, S_{k}\right)=\sqrt{2} R_{0}^{\mathrm{HL}}+\frac{\delta_{R} \sqrt{2}}{4}\left(S_{i}+S_{k}\right),
\end{aligned}
$$

where the quantities $R_{0}^{\mathrm{HL}}$ and $\delta_{R}$ are the equilibrium distance of HS-LS configuration and the lattice parameter misfit of two nn sites, between the HS and LS states, respectively. They are obtained as a function of the nn equilibrium distances $R_{0}^{\mathrm{HH}}=$ $R_{0}(+1,+1), R_{0}^{\mathrm{HL}}=R_{0}(+1,-1)$, and $R_{0}^{\mathrm{LL}}=R_{0}(-1,-1)$ as follows:

$$
\begin{aligned}
R_{0}^{\mathrm{HL}} & =\frac{R_{0}^{\mathrm{HH}}+R_{0}^{\mathrm{LL}}}{2}, \\
\delta_{R} & =\left(R_{0}^{\mathrm{HH}}-R_{0}^{\mathrm{LL}}\right) .
\end{aligned}
$$

The model Hamiltonian is solved using a Monte Carlo (MC) procedure executed on the spin and lattice systems, as follows. First, a spin is randomly selected among the $N^{2}$ sites of the lattice and its initial energy is calculated, then its spin state is flipped and its final energy is calculated. Next, a usual Monte Carlo Metropolis algorithm is used to decide for the final state. Subsequently, we proceed to a second MC process, applied on the sites positions in order to relax mechanically the lattice. For that, we visit sequentially or randomly each lattice site that we move from its initial position by a small displacement $\mu\left(\ll R_{0}^{\mathrm{LL}}\right)$. Afterwards, we calculate the difference between final and the initial elastic energy and we perform a MC process. This procedure can be repeated several times or each 
spin flip in order to reach the equilibrium from both spin and elastic degrees of freedom. The system is warmed up from $T=5$ to $200 \mathrm{~K}$ in steps of increment $1 \mathrm{~K}$, and then cooled down to the initial temperature.

In view of the complex structure of the Hamiltonian of the nanocomposite, in which spin and distortion variables are intricate, which prevents any possible analytical resolution, we use the MC technique to study its thermal properties. The simulations are performed on the square lattice by updating both spin and lattice position variables, using a sequential procedure. The stochastic algorithm is performed in the following way [83]: for a site $i$, randomly selected, with spin $S_{i}= \pm 1$ and position $\vec{r}_{i}$, a new spin value $S_{i}^{\prime}=-S_{i}$ is set without position change. This spin change is accepted or rejected by the usual Metropolis criterion. Once the new spin value is accepted, the lattice is relaxed mechanically by a slight motion of nodes (selected randomly) with a quantity $\mu=0.001 \mathrm{~nm}$, which is much smaller than the average distance between two neighboring sites $(\sim 1 \mathrm{~nm})$. The procedure of the lattice relaxation is repeated 10 times for each spin flip. Afterwards, a new site will be selected randomly, and so on. Once all the nodes of the lattice are visited for the spin change, we define such a step as the unit of the Monte Carlo step and denote it "MCS." In the present simulations, the thermal properties are calculated. At each temperature, we perform $10^{5}$ MCS to reach the equilibrium state and we use 1000 other MCS for the statistics. Within this procedure, each site is displaced $10 \times N_{\text {tot }}^{2}$ times for 1 MCS. So, at each temperature, each spin state and lattice position are updated $\sim 10^{5}$ and $10^{6} \times N_{\text {tot }}^{2}$, times, respectively. We have checked that increasing the simulation time does not affect the final results, which ensures that we reached the stationary state for spin and lattice position variables.

\section{RESULTS AND DISCUSSIONS}

\section{A. Thermal properties of the uncoated core}

The first part of this study starts with the investigations on the bare SCO core as a function of temperature for different sizes, going from $10 \times 10$ to $40 \times 40$, as indicated in Fig. $2($ a). The parameter values of the model $\Delta_{\mathrm{C}}=450 \mathrm{~K}, g=150$, $A_{\mathrm{C}}=B_{\mathrm{C}}=10^{5} \mathrm{~K} \mathrm{~nm}^{-2}$ are chosen so as to reach the behavior of a first-order thermal transition with relatively small sizes. The LS and HS, lattice parameter distances, which should not be confused with the Fe-ligand distances of the molecular coordination sphere, are chosen to be equal to $R_{0}^{\mathrm{LL}}=1 \mathrm{~nm}$ and $R_{0}^{\mathrm{HH}}=1.05 \mathrm{~nm}\left(R_{\mathrm{HL}}=\frac{R_{\mathrm{HH}}+R_{\mathrm{LL}}}{2}=1.025 \mathrm{~nm}\right)$ in good agreement with structural experimental data [84], which report relative lattice expansions of $\sim 1 \%-5 \%$, depending on the molecular crystal packing (presence of pi-pi strong $\pi-\pi$ stacking, "weak" hydrogen-hydrogen bonding, etc.). Although we consider here an isotropic deformation of the crystal lattice, elastic constraints imposed by the interactions between ligands of adjacent molecules who have extended spatial occupations [85-87], may lead to anisotropic unit cell deformations as observed in several SCO systems and reported in recent specific theoretical investigations $[88,89]$. So, the chosen values of the elastic constants, lead to an estimated average bulk modulus

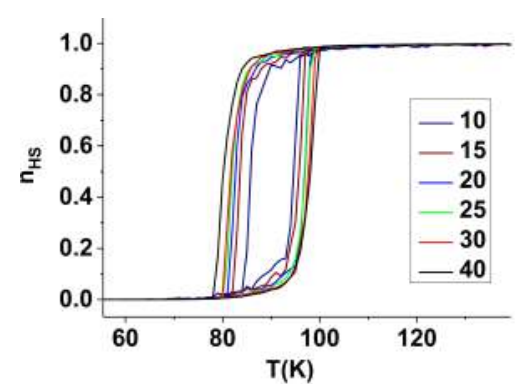

(a)

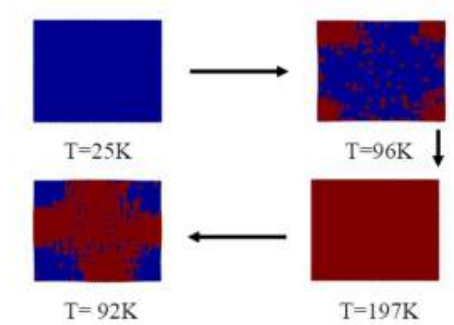

(b)

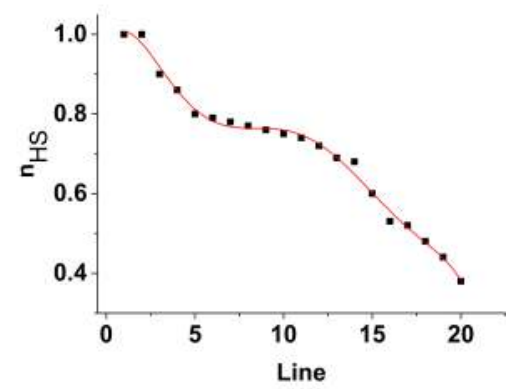

(c)

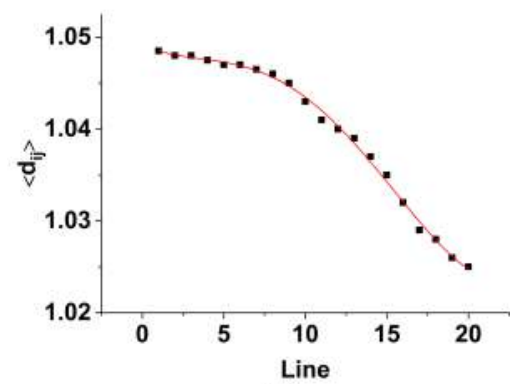

(d)

FIG. 2. (a) Thermal variation of the HS fraction of the bare 2D nanoparticle (with square symmetry) for different sizes, showing the occurrence of a first-order transition and the increase of the thermal hysteresis width with the nanoparticle size. (b) Snapshots showing the spatial distribution of the HS fraction along the thermal hysteresis for the particle $40 \times 40$. (c) and (d) The average HS fraction $n_{\mathrm{HS}}$ and nn distance $\left\langle d_{i j}\right\rangle$ over concentric square perimeters (the center is that of the lattice) as a function of their distance from the center of the nanoparticle for the configuration $T=92 \mathrm{~K}$ of (b). The parameter values are given in the text. (b) The spatial distribution of the HS fraction along the heating and cooling branches of the thermal hysteresis of a bare particle of size $40 \times 40$. It depicts the usual domain nucleation from the corners as a result of surface effects. Here the nucleation cannot start from the center due to the excessive energy barrier that faces the growth of the stable phase inside the metastable one. This behavior is recalled to make easier the comparison with the nanocomposite behavior which will be investigated in the next section. 
$G \approx \frac{A_{\mathrm{C}}}{R_{0}} \sim 10 \mathrm{GPa}$, which is in excellent agreement with available experimental data of Brillouin scattering performed on the single crystal of $\left[\mathrm{Fe}(\mathrm{ptz})_{6}\right]\left(\mathrm{ClO}_{4}\right)_{2}$ which led to bulk modulus values found in the range 5-20 $\mathrm{GPa}[90,91]$. In addition, Fig. 2(a) shows that increasing the size of the core results in an increase of the width of the thermal hysteresis, due the increase of the lifetime of the metastable states, which are stabilized by the elastic long-range interactions between the SCO particles. The transition temperature is here simply given by the relation $T_{\mathrm{eq}}=\frac{\Delta \mathrm{C}}{k_{B} \ln g} \sim 90 \mathrm{~K}$, which is in excellent agreement with the results of MC simulations of Fig. 2(a).

To improve the readability of the present results on the boundary effects, we calculate the average magnetization (or HS fraction) and the average nn distance $\left\langle d_{i j}\right\rangle$ over concentric square perimeters of side $2 \ell+1$ [where $\ell$ goes from 0 to $(N-1) / 2$ ], which are then plotted as a function of their distance $(=\ell)$ from the center of the nanoparticle. The obtained results, summarized in Figs. 2(c) and 2(d) for the temperature $T=92 \mathrm{~K}$ on cooling, for which the corresponding spin state distribution is given in Fig. 2(b). At first sight, one can remark the effect of the boundary conditions on the HS fraction which remains HS in the center and decreases towards that of the LS as one moves away from the center of the lattice. This behavior is also confirmed by the spatial dependence of the average nn distance, which also remains equal to that of the HS state in the bulk and decreases following a sigmoidal trend towards $R_{0}^{\mathrm{LL}}$ when we approach the surface, thus highlighting the boundary effects.

\section{B. Case of active core and shell spin-crossover nanoparticles}

Now we turn to the case of the spin-crossover nanocomposite, schematically described in Fig. 1(a). For that we work on a 2D lattice with a core size $40 \times 40$, with the same model parameters as those of the uncoated core of Fig. 2. This core is now decorated by a spin-crossover shell, with a ligand field $\Delta_{\mathrm{S}}=200 \mathrm{~K}$ with the same degeneracy ratio as that of the core $(g=150)$, whose width is varied from 0 to 5 layers. The used Monte Carlo procedures to solve the thermodynamic properties of this composite system are the same as those used for the uncoated core. The results of the simulations are summarized in Figs. 3-6. First, Fig. 3 reports the thermal variation of the total HS fraction of the nanocomposite for various shell widths. We see that, due to the difference of ligand fields of the core and the shell of the nanocomposite, a two-step transition behavior is obtained, with transition temperatures which are constrained between that of the uncoated core $\left(T_{\mathrm{eq}}^{\text {Core }}=\frac{\Delta_{\mathrm{C}}}{k_{B} \ln g} \approx 90 \mathrm{~K}\right)$ and that of a free shell $\left(T_{\mathrm{eq}}^{\text {Shell }}=\frac{\Delta_{\mathrm{s}}}{k_{B} \ln g} \approx 40 \mathrm{~K}\right)$.

The results show that the two-step transition character is enhanced when we increase the thickness of the shell, leading to a synergy between the two components of the SCO nanocomposite which affects the transition of the whole system. Indeed, the spin transitions appearing in Fig. 3 shift to lower temperatures as a function of shell thickness. Consequently, the value of the HS fraction in the plateau region increases linearly with the ratio shell/core. This is due to the fact that the low-temperature transition (around $50 \mathrm{~K}$ ) concerns exclusively the shell part (which has a lower ligand field value), while the high-temperature transition (around $100 \mathrm{~K}$ ) relates to
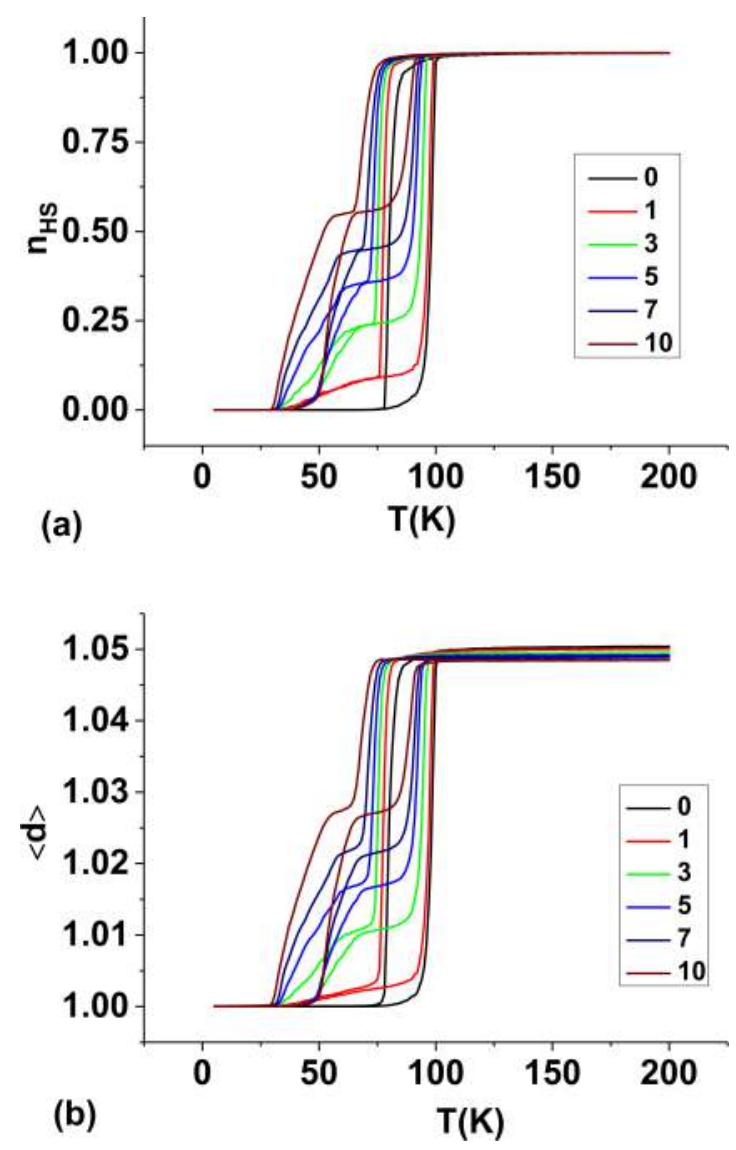

FIG. 3. Thermal variation of the total HS fraction (a) and the average lattice parameter (b) of the SCO nanocomposite for different shell thicknesses for a core size $40 \times 40$. The parameter values are $\Delta_{\mathrm{S}}=200 \mathrm{~K}, T_{\mathrm{eq}}^{\text {Shell }} \approx 40 \mathrm{~K}, \Delta_{\mathrm{C}}=450 \mathrm{~K}, T_{\mathrm{eq}}^{\text {Core }} \approx 90 \mathrm{~K}$. The used values of the elastic constants are $A_{\mathrm{C}}=A_{\mathrm{S}}=10^{5} \mathrm{~K} \mathrm{~nm}^{-2}$ for $\mathrm{nn}$ interactions and $B_{\mathrm{C}}=B_{\mathrm{S}}=10^{5} \mathrm{~K} \mathrm{~nm}^{-2}$ for the nnn interactions in the core and the shell. The equilibrium lattice parameter values were $R_{0}^{\mathrm{HH}}=1.05 \mathrm{~nm}, R_{0}^{\mathrm{LL}}=1 \mathrm{~nm}$, and $R_{0}^{\mathrm{HL}}=1.025 \mathrm{~nm}$ for both constituents.

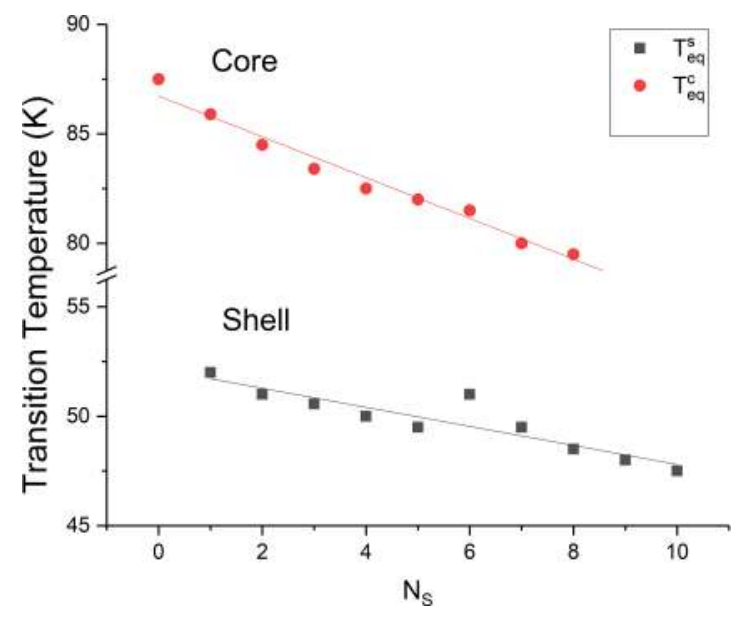

FIG. 4. Average equilibrium transition temperatures of the shell and the core components of the nanocomposite deduced from MC simulations of Fig. 3. Bold lines are the best linear fitting. 

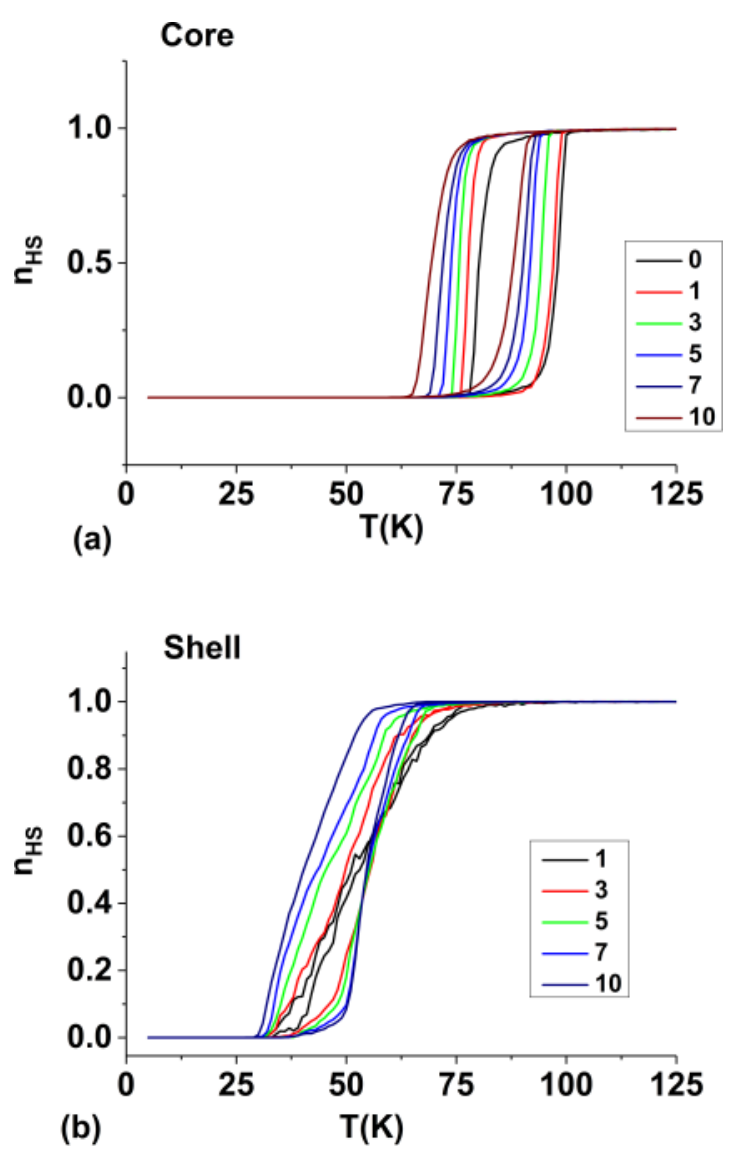

FIG. 5. Thermal response of the HS fraction of the core (a) and shell (b) of the SCO nanocomposite, for different shell thicknesses (from $N_{\mathrm{s}}=0$ to 10 ), corresponding to the results of Fig. 3.

the core. Interestingly, the equilibrium transition temperatures, calculated by $\mathrm{MC}$ as the average value between the upper and lower transition temperatures, behave almost linearly with the shell width, with however a bigger slope $\left(\frac{d T_{\text {eq }}^{\text {core }}}{d N_{\mathrm{S}}}=-1 \mathrm{~K}\right)$, for the core which is more affected by the shell thickness than the shell part $\left(\frac{d T_{\text {cqull }}}{d N_{\mathrm{S}}}=-0.25 \mathrm{~K}\right)$. This is attributed to the difference in the elastic configurations of the two systems. Indeed, while the core is completely surrounded by the HS shell which disfavors its transition from LS to HS or (HS to LS), the shell experiences the opposite situation, since, during its transition from LS to HS (HS to LS), the core is always in the LS (HS). Moreover, the shell has a free surface, which helps the relaxation of the elastic energy excess. Overall, these aspects lead to a different behavior for the core and the shell with temperature.

It is interesting to notice that a spin-crossover core surrounded by an inert matrix, a situation that we have already studied in the past [35], for core-shell spin crossover and prussian blue analogs, leads to a totally different behavior: the transition temperature of the core may increase or decrease, depending on the sign of the lattice parameter misfit between the core and the shell as a result of the elastic effect of the shell which exerts a compressive or a tensile stress on the core.
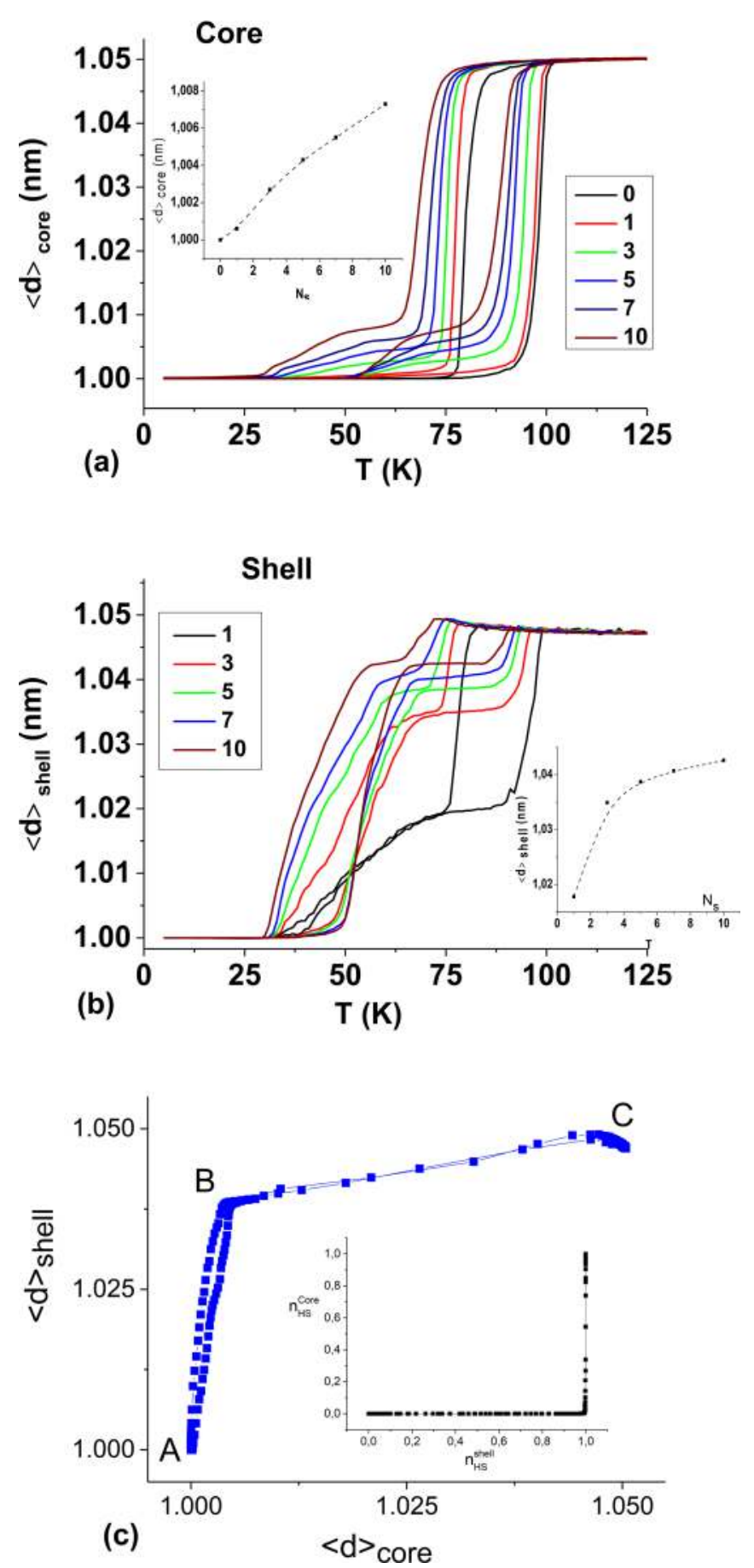

FIG. 6. Thermal dependence of the average nearest-neighbors distance of (a) the core $\langle d\rangle_{\text {core }}$ and (b) the shell $\langle d\rangle_{\text {shell }}$, for different shell thickness values. Insets represent the average nn distance in the plateau region as a function of the shell's thickness. (c) The thermal dependence of the whole core-shell system in the phase space $\left(\langle d\rangle_{\text {core }},\langle d\rangle_{\text {shell }}\right)$ where the temperature is a hidden variable. The associated inset displays the HS fraction of the core vs that of the shell. The parameter values are the same as those of Fig. 3 .

\section{Electronic properties of the SCO nanocomposite}

To get more insights on the synergy between the two SCO subsystems, we have plotted the thermal behavior of the average value of the HS fraction of the core $n_{\mathrm{HS}}^{\text {Core }}$ and 
the shell $n_{\mathrm{HS}}^{\text {Shell }}$ for different shell thicknesses. The results are summarized in Figs. 5(a) and 5(b). Let us first examine the case of the core [Fig. 5(a)]. It is easy to see that the whole hysteresis of the core shifts to lower temperature by increasing the shell thickness. This behavior is consistent with the fact that on heating (cooling), the LS (HS) core takes benefit from the HS (LS) state of the shell which then drives its transition at lower temperature due to the elastic field induced by the shell on the core which expands (contracts) the core network. This expansion/contraction of the core causes an additional local pressure on each site, which then changes its ligand field.

Figure 5(b) is slightly different. According to the results of Sec. II, we can easily deduce that a free shell (a shell without core) makes the spin transition around $T_{\text {eq }}^{\text {shell }}=\frac{\Delta_{\mathrm{S}}}{k_{B} \ln g} \sim 40 \mathrm{~K}$. This transition gives a thermal hysteresis for thick shells and the transition is gradual for thin shells. When reducing the size of the shell, the latter feels the effect of the core. Thus, on heating (cooling) the shell from the LS (HS) state, the switching to HS (LS) is hindered by the LS (HS) state of the core, which "opposes to" the expansion (contraction) of the shell. This elastic strain effect leads to increase the effective ligand field of the shell, which results in the increase of its transition temperature, as shown in Fig. 4.

So, in the heating regime, the shell switches first from LS to HS while the core remains in the LS state. Compared to a free infinite shell, whose transition temperature is $T_{\text {eq }}^{\text {shell }}=$ $\frac{\Delta_{\mathrm{S}}}{k_{B} \ln (g)} \approx 40 \mathrm{~K}$, the shell transition temperature of relatively thin shells in the nanocomposite is higher [see Fig. 5(b)] and the thermal transitions quite gradual. This is due to the pressure exerted by the core, which prevents the formation of single domain nucleation. Increasing the shell thickness reduces the effect of the core, which enhances the cooperative character of the shell, leading to the decrease of its transition temperature towards that of the infinite shell. The latter effect leads to the emergence of an associated thermal hysteresis. We should mention that in this study we considered the mechanical properties of the core and the shell as equal, which means that we use the same elastic constants for both elastic networks.

\section{Mechanical properties of the SCO nanocomposite}

We now focus on the thermal dependence of the elastic properties of the SCO nanocomposite during the transition. For that we have first carefully inspected the mechanical properties of the whole lattice, by plotting the thermal behavior of the lattice parameter, given in Fig. 3(b), which are determined concomitantly with the results of HS fraction, shown in Fig. 3(a). We calculated the thermal dependence of the average lattice parameter over the total lattice and it follows a similar trend as that of the total HS fraction given in Fig. 3(a), with a clear existence of the two-step transitions character for increased shell thickness. Following the same idea as for the electronic properties, we now inspect the thermal dependence of the core and shell lattice parameters and represent their thermal behaviors in Figs. 6(a) and 6(b). Interestingly, comparing the mechanical responses of the core and the shell in Figs. 6(a) and 6(b) for $N_{\mathrm{s}}=5$ (for example), shows that on heating (cooling) the lattice parameter of the core (shell) starts to adapt to the change of that of the shell (core) before $T=50 \mathrm{~K}$, while in this temperature range, its corresponding HS fraction is still insensitive to this change, as clearly depicted in the associated Figs. 5(a) and 5(b). This behavior undoubtedly emphasizes the crucial role of the mechanical coupling between the two subsystems in the resulting thermal behavior of the whole system.

Now we wish to pay attention to the values of the lattice parameter. Whatever the thickness of the shell, all lattice parameters in Fig. 6 change between the values of 1.0 and $1.05 \mathrm{~nm}$ corresponding to those of the LS and HS states, respectively. However, it is interesting to understand the values of the relaxed lattice parameters of the shell and the core in the plateau region, which derive directly from antagonist interactions among the core and the shell and inside them. The forthcoming section sheds a better light on the relevant physical parameters governing this aspect, by employing a simple analysis to predict them with respect to the shell thickness.

To highlight the elastic interaction between the core and the shell, we follow the behavior of the whole system by plotting in Fig. 6(c) the dependence of the average lattice parameter of the core $\langle d\rangle_{\text {core }}$ as a function of that of the shell $\langle d\rangle_{\text {shell }}$, while in the inset of the figure, we report the dependence of the HS fraction of the core $n_{\mathrm{HS}}^{\text {Core }}$ vs that of the shell $n_{\mathrm{HS}}^{\text {Shell }}$ during the thermal transitions for the case of a shell width $\mathrm{N}_{\mathrm{s}}=5$. Remarkably, the two HS fractions, depicted in the inset of Fig. 6(c), appear to be totally independent, since the HS fraction of the core starts to change only when all shell is converted to the HS state. In contrast, Fig. 6(c) demonstrates that there is a strong correlation between the structural evolutions of the shell and core lattice parameters. Indeed, the lattice parameter of the core is impacted by the change of that of the shell at the beginning. Accordingly, between point $\mathrm{A}$, for which $n_{\mathrm{HS}}^{\text {Shell }}=$ 0 and $n_{\mathrm{HS}}^{\text {Core }}=0$, and point $\mathrm{B}$, for which have $n_{\mathrm{HS}}^{\text {Shell }}=1$ and $\mathrm{n}_{\mathrm{HS}}^{\text {Core }}=0$, we can easily see that the shell (the core) experiences a positive (negative) pressure since $\langle d\rangle_{\text {shell }}<R_{0}^{\mathrm{HH}}\left(\langle d\rangle_{\text {core }}>\right.$ $\left.R_{0}^{\mathrm{LL}}\right)$. Thus, in the first regime where the shell converts from LS to HS, the shell's (core's) lattice parameter reaches the value $1.037 \mathrm{~nm}(1.0047 \mathrm{~nm})$ instead of $1.05 \mathrm{~nm}(1.0 \mathrm{~nm})$. This nonuniform distribution of compressive strain inside the shell and tensile strain inside the core is the result of the retroaction of the core to the spin conversion of the shell. Between points $\mathrm{B}$ and $\mathrm{C}$, the core is then converted in the HS state from both electronic and elastic degrees of freedom. In this regime, where the main elastic changes occur in the core, the latter drives the shell which finishes its elastic conversion.

\section{E. Analytical expressions of the relaxed lattice parameters}

To tackle the behavior of the elastic properties of the model, which monitor those of the HS fraction, we study analytically the shell-thickness dependence of the mechanically relaxed lattice parameters of the core and the shell parts of the nanocomposite.

It is worth mentioning that the interplay between the elastic properties of the core and the shell SCO parts is an interesting, but a bit complex, problem whose exact analytical treatment is out of reach. By construction, the lattice parameters of the core and the shell are equal to $R_{0}^{\mathrm{LL}}$ and $R_{0}^{\mathrm{HH}}$ in the $\mathrm{LS}$ and HS states, respectively. So, in these two states, the system is perfectly relaxed. However, in the plateau region, which 
TABLE I. Number of nn and nnn distances of the core, shell, and interface that are used to evaluate the analytical expression of the elastic energy of Eq. (7).

\begin{tabular}{lccc}
\hline \hline Number of bonds & Core & Shell & Interface \\
\hline nn bonds & $N_{\mathrm{C}}^{\mathrm{nn}}=2 \times N_{\mathrm{C}}\left(N_{\mathrm{C}}-1\right)$ & $N_{\mathrm{S}}^{\mathrm{nn}}=2 \times N \times(N-1)-N_{\mathrm{C}}^{\mathrm{nn}}-N_{\mathrm{int}}^{\mathrm{nn}}$ & $N_{\mathrm{int}}^{\mathrm{nn}}=4 \times N_{\mathrm{C}}(=0$ if $N \mathrm{~s}=0)$ \\
nnn bonds & $N_{\mathrm{C}}^{\mathrm{nnn}}=2 \times\left(N_{\mathrm{C}}-1\right)^{2}$ & $N_{\mathrm{S}}^{\mathrm{nnn}}=2 \times(N-1)^{2}-N_{\mathrm{C}}^{\mathrm{nnn}}-N_{\mathrm{int}}^{\mathrm{nnn}}$ & $N_{\text {int }}^{\mathrm{nnn}}=8 \times N_{\mathrm{C}}(=0$ if $N \mathrm{~s}=0)$ \\
\hline \hline
\end{tabular}

corresponds to a core in the LS state and a shell in the HS state (from the electronic point of view), the respective relaxed lattice parameters of the core and the shell are not those of the pure LS and HS states, as demonstrated by MC simulations in the previous section.

Here we develop an approximate method aiming to evaluate the analytical expressions of the relaxed lattice parameters of the core and the shell. So, the strategy is to consider uniform instantaneous nn distances $x_{\mathrm{c}}, x_{\mathrm{s}}$, and $x_{i}$ for the core, shell, and interface, respectively. Then we count exactly the number of $n n$ and nnn bonds associated with each of these three areas of the nanocomposite. These expressions are summarized in Table I. This information is then used to evaluate the elastic energy, allowing the finding of the equilibrium distances on one hand, and the total energy (elastic + electronic) to express the transition temperatures on the other hand.

In Table I $N=N_{\mathrm{C}}+2 N_{\mathrm{S}}$ represents the total number of atoms along the edge of the core-shell system. On the other hand, the total numbers of atoms in the composite is $N^{2}=\left(N_{\mathrm{C}}+2 N_{\mathrm{s}}\right)^{2}$, while inside the core and the shell they are respectively equal to $N_{\mathrm{C}}^{2}$ and $4 N_{\mathrm{s}}\left(N_{\mathrm{s}}+N_{\mathrm{C}}\right)$.

Next, we determine the expression of the total elastic energy of the lattice in the plateau region, knowing that the total elastic energies in the pure LS and HS states are equal to zero. So, as previously stated, we consider a $2 \mathrm{D}$ homogeneous lattice with the uniform average lattice parameter $x$ in the core, shell, and interface. On the other hand, in the plateau region, the equilibrium distances between core nn (nnn) is equal to $R_{0}^{\mathrm{LL}}$ ( $R_{0}^{\mathrm{LL}} \sqrt{2}$ ), that of the shell is $R_{0}^{\mathrm{HH}}\left(R_{0}^{\mathrm{HH}} \sqrt{2}\right)$, and that of the sites located at the interface is $R_{0}^{\mathrm{HL}}\left(R_{0}^{\mathrm{HL}} \sqrt{2}\right)$. The instantaneous and equilibrium nn and nnn distances of the core, shell, and interface are summarized in Table II.

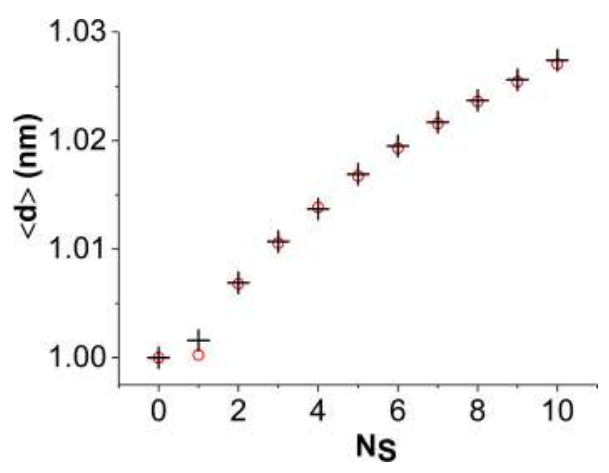

FIG. 7. Average lattice parameter $\langle d\rangle$ in the plateau region as a function of the shell's thickness. Black crosses are the results of the MC simulations data and open red circles are the prediction of the analytical treatment, derived from Eq. (8).
Using the data of Tables I and II, one can easily calculate the total elastic energy in the plateau region, whose expression is given by

$$
\begin{aligned}
E_{\text {elas }}= & \left(\frac{A}{2} N_{\mathrm{c}}^{\mathrm{nn}}+B N_{\mathrm{c}}^{\mathrm{nnn}}\right)\left(x-R_{0}^{\mathrm{LL}}\right)^{2} \\
& +\left(\frac{A}{2} N_{\mathrm{s}}^{\mathrm{nn}}+B N_{\mathrm{s}}^{\mathrm{nnn}}\right)\left(x-R_{0}^{\mathrm{HH}}\right)^{2} \\
& +\left(\frac{A}{2} N_{i}^{\mathrm{nn}}+B N_{\mathrm{i}}^{\mathrm{nnn}}\right)\left(x-R_{0}^{\mathrm{HL}}\right)^{2} .
\end{aligned}
$$

Minimizing the elastic energy Eq. (7) with respect to the average lattice parameter $x$ gives the average relaxed lattice parameter $x_{\text {relax }}$ as follows:

$$
\begin{aligned}
x_{\text {relax }}= & \frac{1}{A N_{\mathrm{tot}}^{\mathrm{nn}}+2 B N_{\mathrm{tot}}^{\mathrm{nnn}}}\left[\left(A N_{\mathrm{c}}^{\mathrm{nn}}+2 B N_{\mathrm{c}}^{\mathrm{nnn}}\right) R_{0}^{\mathrm{LL}}\right. \\
& \left.+\left(A N_{\mathrm{s}}^{\mathrm{nn}}+2 B N_{\mathrm{s}}^{\mathrm{nnn}}\right) R_{0}^{\mathrm{HH}}+\left(A N_{i}^{\mathrm{nn}}+2 B N_{i}^{\mathrm{nnn}}\right) R_{0}^{\mathrm{HL}}\right],
\end{aligned}
$$

where $N_{\mathrm{tot}}^{\mathrm{nn}}=N_{\mathrm{c}}^{\mathrm{nn}}+N_{\mathrm{s}}^{\mathrm{nn}}+N_{i}^{\mathrm{nn}}$ and $N_{\mathrm{tot}}^{\mathrm{nnn}}=N_{\mathrm{c}}^{\mathrm{nnn}}+N_{\mathrm{s}}^{\mathrm{nnn}}+$ $N_{\mathrm{i}}^{\mathrm{nnn}}$ are the total numbers of $\mathrm{nn}$ and nnn bonds inside the whole lattice.

The shell-thickness dependence of the relaxed parameter $x_{\text {relax }}$ in the plateau region is displayed in Fig. 7 which shows that the relaxed parameter is indeed equal to that of the LS state for a bare (uncoated) core and tends to be closer to that of the HS lattice parameter for thicker shells.

It is legitimate to ask how these $0 \mathrm{~K}$ calculations work so well when compared to MC data, while the lattice relaxation in the MC simulations has been performed at defined temperatures. In fact, in the plateau region, the nanoparticle presents a specific spin configuration (all shell atoms are HS and all core atoms are LS), and the objective was to find the minimum elastic energy of this configuration. The analytical method used here is equivalent to the relaxation of the lattice using deterministic Newton equations in the case of the presence of a strong viscosity. We have introduced this method in Paez et al.

TABLE II. Instantaneous and equilibrium nn and nnn distances of the core, shell, and interface, considered in the analytical calculations, are developed in Eqs. (7) and (8).

\begin{tabular}{lcccc}
\hline \hline & $\begin{array}{c}\mathrm{nn} \\
\text { distance }\end{array}$ & $\begin{array}{c}\mathrm{nnn} \\
\text { distance }\end{array}$ & $\begin{array}{c}\text { nn equilibrium } \\
\text { distance }\end{array}$ & $\begin{array}{c}\text { nnn equilibrium } \\
\text { distance }\end{array}$ \\
\hline Core & $x$ & $x \sqrt{ } 2$ & $R_{0}^{\mathrm{LL}}$ & $R_{0}^{\mathrm{LL}} \sqrt{ } 2$ \\
Shell & $x$ & $x \sqrt{ } 2$ & $R_{0}^{\mathrm{HH}}$ & $R_{0}^{\mathrm{HH}} \sqrt{ } 2$ \\
Interface & $x$ & $x \sqrt{ } 2$ & $1 / 2\left(R_{0}^{\mathrm{LL}}+R_{0}^{\mathrm{HH}}\right)$ & $1 / 2\left(R_{0}^{\mathrm{LL}}+R_{0}^{\mathrm{HH}}\right) \sqrt{ } 2$ \\
\hline \hline
\end{tabular}


[88] and its relevance was also tested recently in Traiche et al. [89] when studying frustrated SCO lattices. The main reason of its efficiency comes from the fact that, when the right spin configuration is fixed, relaxing the lattice using deterministic mechanics or molecular dynamics or Monte Carlo simulations on positions leads to the same average results. However, significant differences will emerge between local and global fluctuations of the average distance (or elastic energy), which are not taken into account in the analytical procedure.

\section{F. Spatial distribution of the HS fraction}

To get more insights about the spatiotemporal character of dynamics of the SCO transition, we monitor the spatial distribution of the HS fraction in the core and shell during the development of the spin states along the thermal hysteresis of the whole lattice [see Fig. 8(a)], obtained for $N_{\mathrm{S}}=5$. Selected snapshots of the electronic state of the system are shown in Fig. 8(b) along the heating and cooling branches of the associated thermal hysteresis. There, the red (blue) dots denote HS (LS) atoms. We observe that on heating, the domain nucleation of the HS phase appears in the shell part of the nanocomposite from the corners and propagates along the width, while the core lattice remains in the LS state, although the global volume of the lattice increases, which leads the core to experience a tensile strain from the shell. This is easily readable on the spatial dependence of the lattice parameter [see Fig. 8(c)] along the $x$ direction for the atomic line located at the coordinate $j=\frac{N}{2}$ in the plateau region. We see that the lattice parameter changes from the value of the HS state (in the shell part) far from the shell-core interface region and then increases when approaching the core-shell interface region (due to the mechanical retroaction of the core) before decreasing abruptly in the core region. However, we remark that in all regions (core and shell) the relaxed lattice parameter remains bigger than its equilibrium values $R_{0}^{\mathrm{HH}}=1.05 \mathrm{~nm}$ in the shell and $R_{0}^{\mathrm{LL}}=1 \mathrm{~nm}$ in the core. Obviously these results depend on the shell thickness and the conclusions drawn here remain qualitatively valid for other sizes.

The tensile stress exerted by the shell on the core is presented in Fig. S1 (in the Supplemental Material) [92] through the plot of the spatial map of the local pressure, calculated on each site of the lattice. In Fig. 8(b), where we report the spatial distribution of the HS and LS states, we can observe a significant change in the growth mode of the HS fraction of the core. Indeed, the transition of the core does not lead to the usual single domain nucleation, in spite of being of first order (due to the existence of a thermal hysteresis). In fact, the shell facilitates the formation of core nucleation sites, which leads the nucleation to start from everywhere, to form a ramified HS structure, as seen in snapshots (D 90).

On the other hand, following the same procedure as in Figs. 2(c) and 2(d), to compare the behavior of the bulk and surface sites, we plot in Fig. 8(d) the average lattice parameter (calculated over concentric square perimeters) as a function of their distance from the center of the core-shell nanoparticle. The obtained results show a continuous increase of the lattice parameter following two regimes: (i) a slow regime in the bulk and (ii) an "explosive" growth starting from the core-shell interface and reaching its maximum at the surface, where the
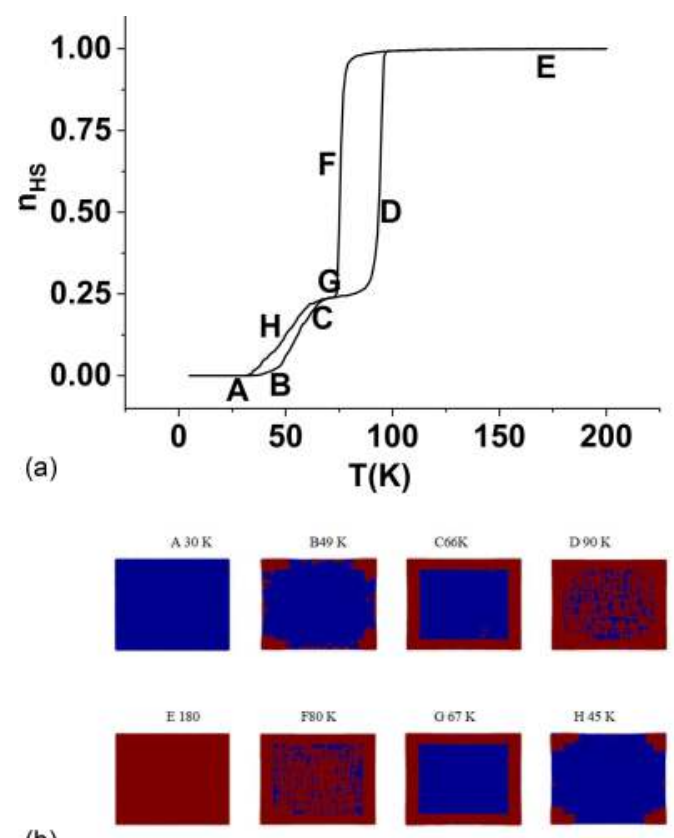

(b)
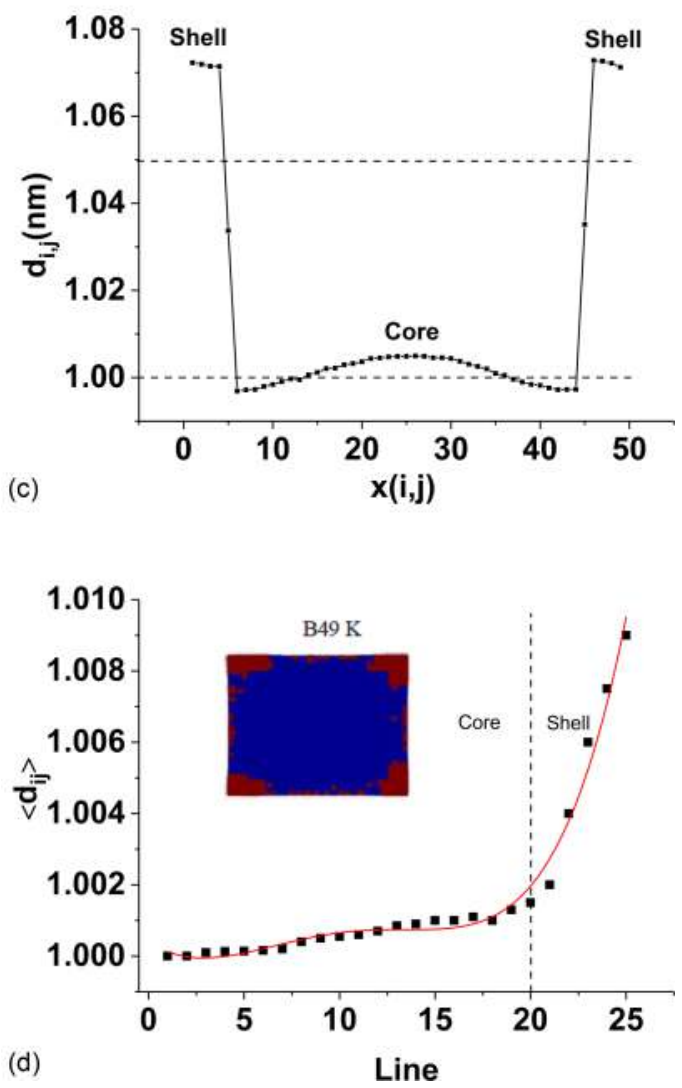

FIG. 8. (a) Temperature dependence of the SCO nanocomposite showing a plateau behavior, obtained for $N_{\mathrm{C}}=40$ and $N_{\mathrm{S}}=5$. (b) HS fraction snapshots showing the spatial distribution of the HS and LS states corresponding to the thermal hysteresis given in (a). (c) Spatial dependence of the nn distance along the $x$ direction in the plateau region of the thermal hysteresis of (a). (d) Average nn distance $\left\langle d_{i j}\right\rangle$ calculated over concentric square perimeters (the center is that of the lattice) as a function of their distance from the center of the core-shell for $T=49 \mathrm{~K}$ on heating. Remark the abrupt increase of the $\mathrm{nn}$ distance near the surface. 


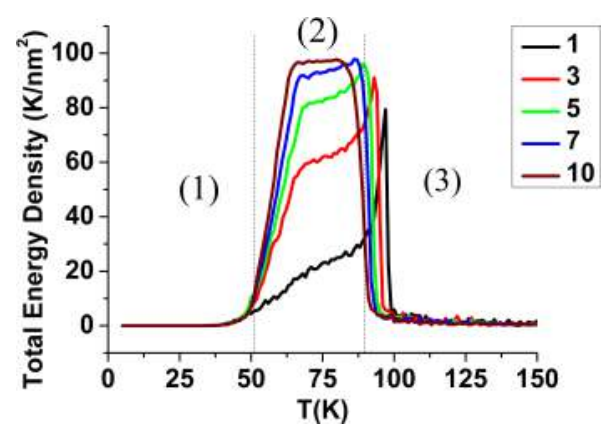

FIG. 9. Thermal variation of the total elastic energy density in the nanocomposite for different shell thicknesses, for the same core size $40 \times 40$, along with the heating branch of the thermal hysteresis.

spin state combined with the open boundary effects allow the interatomic distance to reach its maximum value.

On cooling from the snapshot (E180), the core surrounded by a HS shell switches first to the LS state (G 67). Here the shell prevents mechanically the HS to LS switching of the core. However, the nucleation of the LS state is initiated preferentially in the vicinity of the interface region (F 80), where the LS state experiences less tensile strain due to the retroaction of the core [see Fig. 8(c)]. As a result, the LS domains grow rapidly along the interface region and then propagate towards the center of the lattice.

After the complete transformation of the core, the shell starts to convert from HS to LS from the edge atoms which are favored compared to those of the shell's corners. Indeed, each shell atom situated in the edge connects to three (one nn and two nnn) HS core atoms, while those of the corner connect only to one HS core atom.

\section{G. Elastic energy and spatiotemporal behavior of the nanocomposite at the transition}

To continue in the detailed investigations on the physical mechanisms underlying the previous behaviors, we have first calculated the thermal dependence of the total elastic energy density for different shell thicknesses, the results of which are presented in Fig. 9. The contributions from the core and the shell are summarized in Fig. S2 of the Supplemental Material [92]. First of all, we observe that the thermal behavior of the total elastic energy exhibits a similar behavior as that of the HS fraction since it shows the existence of three regions. On heating, the elastic energy density suddenly increases at the transition temperature of the shell $(\sim 50 \mathrm{~K})$ then its slope smooths significantly in the region $50-90 \mathrm{~K}$, to increase again before $100 \mathrm{~K}$ and to fall down at a higher temperature. These regions [(1), (2), and (3)] fit well with the spatiotemporal behavior of the nanocomposite during the heating process, presented in Fig. 9. We then find that the first abrupt increase of the elastic energy at $50 \mathrm{~K}$ corresponds to the nucleation of the HS fraction in the shell part, causing an increase of the misfit elastic energy. While in the plateau region of Fig. 9, we observe deceleration in the increase of elastic energy, which we attribute to the saturation of the HS state in the shell. However due to the connection of the shell with the core, which is still LS, the former experiences a complex tensile/compressive strain spatially distributed over both parts of the nanocomposite. The presence of these strains creates the plateaulike (second region) in the thermal behavior of the elastic energy. In this region, the thermal fluctuations increase, leading to the transformation of the core.

\section{CONCLUSION}

In conclusion, we have analyzed the thermodynamic properties of a spin-crossover nanocomposite made of two different spin-crossover units in the core-shell configuration. We have seen that this configuration allows an efficient mechanical coupling between the two spin-crossover materials since they influence the thermodynamic of each other. Due to this mechanical coupling, the thermal dependence of the total nanocomposite is far from the sum of each of its constituents. To describe this phenomenon, we have adapted our electroelastic model to the case of a nanocomposite made of two-elastically coupled SCO materials having different transitions temperatures and thermal hysteresis widths. The results showed that according to the difference of transition temperatures and elastic interactions, the nanocomposite may lead to all possibilities of spin transitions: two step, gradual, one step with thermal hysteresis, etc. In the case of a twostep spin transition, with $T_{\mathrm{eq}}^{\text {Shell }}<T_{\mathrm{eq}}^{\text {Core }}$, we found that the transition of the shell part at low temperature does affect significantly the elastic properties of the core (and vice versa), whose lattice parameter starts to change long before any variation of its spin state. We found that the interplay between the electroelastic properties of both constituents leads to a complex distribution of a pressure (or elastic energy) inside the lattice. In practice, we have demonstrated that although the thermal spin transition of the core (in the nanocomposite) is of first order, the growth of HS macroscopic domains is prevented by the presence of the shell, which changes this mechanism to that of homogenous ramified structures, in a similar way as those observed in the Ising models [93].

Finally, the model results are qualitatively comparable with the available experimental results of Wang et al. [14]. Moreover, the quantitative approach to go towards the experimental results should include the size distribution of the nanocomposites (shell/core), the effect of shape $[65,94,95]$, as well as their 3D character [96]. Here we have considered a squareshaped SCO core nanoparticle surrounded by a regular shell width. However, several parameters may affect the switching properties of the nanoparticles. Among them, one may quote: (i) the existence of voids which occurs in nanoparticles as reported by Lacroix et al. [80,81], in magnetic nanoparticles and the presence of microstructural defects. Moreover, as we have emphasized in Oubouchou et al. [58], the stability of the bulk structure for small particles is questionable, as reported in nanoparticles of Co-Pt bimetallic clusters [97] that have shown different structures, depending on their size. In addition, real nanoparticle materials, involve surface relaxation and surface reconstructions, which may play on the geometric features and act as boundary effects, leading to irregular shapes or widths of the shell. On another level, we mention that to our best knowledge, there were no experimental proofs leading to discriminate between these possible causes. Indeed, most of the experimental studies on SCO nanoparticles, if not all, that 
have been realized on a large number of nanoparticles. As long as experimental studies on one single nanoparticle, as it was done in magnetic nanoparticles, are not realized in switchable SCO solids, this issue will still be a matter for debate.

Furthermore, an interesting extension of the current model related to the consideration of $2 \mathrm{D}$ core-shell triangular lattices [61], which do not require the use of nnn interactions to keep their stability is very appealing, especially if we consider antiferromagnetic interactions between the spin states. Their competition with the magnetoelastic interactions which stabilize long-range ferromagnetic order may lead to nontrivial behaviors; a very interesting problem from both elastic and magnetic point of views, deserving to be investigated in the future.

Finally, we would like to emphasize that when the rigidities of the core and the shell are significantly different and require the use of different elastic constants and lattice parameters for the two media, we expect a less efficient core-shell mechanical coupling, due to the existence of an acoustic impedance. The use of SCO materials having different transition temperatures and similar elastic properties is then of interest to generate new multistabilities (two, three, or even more steps), which can be used as q-bit systems for memories. SCO nanocomposites made of several multilayers with different transition temperatures constitute an excellent way to design multistable SCO materials with high tailored performances.

\section{ACKNOWLEDGMENT}

This work was supported by CNRS ("Centre National de la Recherche Scientifique"), the universities of Versailles, Paris Saclay, Algeria USTHB, and the French "Ministère de la Recherche".
[1] E. König, in Nature and Dynamics of the Spin-State Interconversion in Metal Complexes (Springer, Berlin, 1991), pp. 51-152.

[2] P. Gutlich, Spin crossover in iron(II)-complexes, Struct. Bond. 44, 83 (1981).

[3] S. Decurtins, P. Gutlich, C. P. Kohler, H. Spiering, and A. Hauser, Light-induced excited spin state trapping in a transition-metal complex - the hexa-1-propyltetrazole-iron (II) tetrafluoroborate spin-crossover system, Chem. Phys. Lett. 105, 1 (1984).

[4] H. Toftlund, Spin equilibria in iron(II) complexes, Coord. Chem. Rev. 94, 67 (1989).

[5] C. P. Köhler, R. Jakobi, E. Meissner, L. Wiehl, H. Spiering, and P. Gütlich, Nature of the phase transition in spin crossover compounds, J. Phys. Chem. Solids 51, 239 (1990).

[6] M. A. Halcrow, Structure: Function relationships in molecular spin-crossover complexes, Chem. Soc. Rev. 40, 4119 (2011).

[7] O. Kahn, J. Kröber, and C. Jay, Spin transition molecular materials for displays and data recording, Adv. Mater. 4, 718 (1992).

[8] O. Kahn, Spin-crossover molecular materials, Curr. Opin. Solid State Mater. Sci. 1, 547 (1996).

[9] J. Zarembowitch and O. Kahn, Spin transition molecular systems; towards information storage and signal processing, New J. Chem. 15, 181 (1991).

[10] J. Linares, E. Codjovi, and Y. Garcia, Pressure and temperature spin crossover sensors with optical detection, Sensors 12, 4479 (2012).

[11] Y. Garcia, P. J. vanKoningsbruggen, E. Codjovi, R. Lapouyade, O. Kahn, and L. Rabardel, Non-classical Fe-II spin-crossover behaviour leading to an unprecedented extremely large apparent thermal hysteresis of $270 \mathrm{~K}$ : Applicationfor displays, J. Mater. Chem. 7, 857 (1997).

[12] O. Kahn and C. J. Martinez, Spin-transition polymers: From molecular materials toward memory devices, Science 279, 44 (1998).

[13] R.-J. Wei, Q. Huo, J. Tao, R.-B. Huang, and L.-S. Zheng, Spin-crossover $\mathrm{Fe}^{\mathrm{II}}{ }_{4}$ squares: Two-step complete spin transition and reversible single-crystal-to-single-crystal transformation, Angew. Chem. Int. Ed. 50, 8940 (2011).

[14] Y.-X. Wang, D. Qiu, S.-F. Xi, Z.-D. Ding, Z. Li, Y.Li, X. Ren, and Z.-G. Gu, Iron(II)-triazole core-shell nanocomposites: Toward multistep spin crossover materials, Chem. Commun. 52, 8034 (2016).

[15] C. Jureschi, J. Linares, A. Rotaru, and Y. Garcia, Multi-sin 3D spin crossover nanoparticles simulated by an Ising model using entropic sampling Monte Carlo technique, Magnetochemistry 2 , 13 (2016).

[16] D. Chiruta, C.-M. Jureschi, J. Linares, P. R. Dahoo, Y. Garcia, and A. Rotaru, On the origin of multi-step spin transition behaviour in 1D nanoparticles, Eur. Phys. J. B 88, 233 (2015).

[17] E. Milin, S. Belaïd, V. Patinec, S. Triki, G. Chastanet, and M. Marchivie, Dinuclear spin-crossover complexes based on tetradentate and bridging cyanocarbanion ligands, Inorg. Chem. 55, 9038 (2016).

[18] T. Kosone, I. Tomori, C. Kanadani, T. Saito, T. Mochida, and T. Kitazawa, Unprecedented three-step spin-crossover transition in new 2-dimensional coordination polymer $\{\mathrm{FeII}(4-$ methylpyridine $\left.)_{2}\left[\mathrm{AuI}(\mathrm{CN})_{2}\right]_{2}\right\}$, Dalton Trans. 39, 1719 (2010).

[19] M. Nihei, H. Tahira, N. Takahashi, Y. Otake, Y. Yamamura, K. Saito, and H. Oshio, Multiple bistability and tristability with dual spin-state conversions in $\left[\mathrm{Fe}(\mathrm{dpp})_{2}\right]\left[\mathrm{Ni}(\mathrm{mnt})_{2}\right]_{2} \cdot \mathrm{MeNO}_{2}$, J. Am. Chem. Soc. 132, 3553 (2010).

[20] A. Kaiba, H. J. Shepherd, D. Fedaoui, P. Rosa, A. E. Goeta, N. Rebbani, J. F. Létard, and P. Guionneau, Crystallographic elucidation of purely structural, thermal and light-induced spin transitions in an iron(II) binuclear complex, Dalton Trans. 39, 2910 (2010).

[21] M. Seredyuk, K. O. Znovjyak, J. Kusz, M. Nowak, M. C. Muñoz, and J. A. Real, Control of the spin state by charge and ligand substitution: Two-step spin crossover behaviour in a novel neutral iron(II) complex, Dalton Trans. 43, 16387 (2014).

[22] H. P.Zhang, L. C. Yang, L. J. Fu, Q. Cao, D. L. Sun, Y. P. Wu, and R. Holze, Core-shell structured electrode materials for lithium ion batteries, J. Solid State Electrochem. 13, 1521 (2009).

[23] L. Zhi-Hua, W. Yu-Xia, Q. Dan, L. Zai-Jun, and G. Zhi-Guo, AAO assisted 1D confined assembly and 2D surface filming of iron(II) triazole nanomaterial and spin-crossover properties, Chinese J. Inorg. Chem. 33, 2311 (2017).

[24] G. Félix, W. Nicolazzi, L. Salmon, G. Molnár, M. Perrier, G. Maurin, J. Larionova, J. Long, Y. Guari, and A. Bousseksou, 
Enhanced Cooperative Interactions at the Nanoscale in SpinCrossover Materials with a First-Order Phase Transition, Phys. Rev. Lett. 110, 235701 (2013).

[25] H. J. Shepherd, G. Molnar, W. Nicolazzi, L. Salmon, and A. Bousseksou, Spin crossover at the nanometre scale, Eur. J. Inorg. Chem. 2013, 653 (2013).

[26] A. Bousseksou, G. Molnar, L. Salmon, and W. Nicolazzi, Molecular spin crossover phenomenon: Recent achievements and prospects, Chem. Soc. Rev. 40, 3313 (2011).

[27] A. Rotaru, F. Varret, A. Gindulescu, J. Linares, A. Stancu, J. F. Letard, T. Forestier, and C. Etrillard, Size effect in spin-crossover systems investigated by FORC measurements, for surfacted $\left[\mathrm{Fe}\left(\mathrm{NH}_{2} \text {-trz }\right)_{3}\right](\mathrm{Br})_{2} \cdot 3 \mathrm{H}_{2} \mathrm{O}$ nanoparticles: $\mathrm{Re}$ versible contributions and critical size, Eur. Phys. J. B 84, 439 (2011)

[28] A. Carné, C. Carbonell, I. Imaz, and D. Maspoch, Nanoscale metal-organic materials, Chem. Soc. Rev. 40, 291 (2011).

[29] R. M. van der Veen, O. H. Kwon, A. Tissot, A. Hauser, and A. H. Zewail, Single-nanoparticle phase transitions visualized by four-dimensional electron microscopy, Nat. Chem. 5, 395 (2013).

[30] L. Catala, D. Brinzei, Y. Prado, A. Gloter, O. Stéphan, G. Rogez, and T. Mallah, Core-multishell magnetic coordination nanoparticles: Toward multifunctionality on the nanoscale, Angew. Chem. Int. Ed. 48, 183 (2009).

[31] A. C. Felts, M. J. Andrus, E. S. Knowles, P. A. Quintero, A. R. Ahir, O. N. Risset, C. H. Li, I. Maurin, G. J. Halder, K. A. Abboud, M. W. Meisel, and D. R. Talham, Evidence for interfaceinduced strain and its influence on photomagnetism in prussian blue analogue core-shell heterostructures, $\mathrm{Rb}_{a} \mathrm{Co}_{b}\left[\mathrm{Fe}(\mathrm{CN})_{6}\right]_{c}$. $m \mathrm{H}_{2} \mathrm{O} @ \mathrm{~K}_{j} \mathrm{Ni}_{k}\left[\mathrm{Cr}(\mathrm{CN})_{6}\right]_{l} \cdot n \mathrm{H}_{2} \mathrm{O}$, J. Phys. Chem. C 120, 5420 (2016).

[32] D. M. Pajerowski, M. J. Andrus, J. E. Gardner, E. S. Knowles, M. W. Meisel, and D. R. Talham, Persistent photoinduced magnetism in heterostructures of prussian blue analogues, J. Am. Chem. Soc. 132, 4058 (2010).

[33] D. M. Pajerowski, B. Ravel, C. H. Li, M. F. Dumont, and D. R. Talham, X-ray absorption study of structural coupling in photomagnetic prussian blue analogue core@shell particles, Chem. Mater. 26, 2586 (2014).

[34] A. C. Felts, A. Slimani, J. M. Cain, M. J. Andrus, A. R. Ahir, K. A. Abboud, M. W. Meisel, K. Boukheddaden, and D. R. Talham, Control of the speed of a light-induced spin transition through mesoscale core-shell architecture, J. Am. Chem. Soc. 140(17), 5814 (2018).

[35] A. Tissot, C. Enachescu, and M. L. Boillot, Control of the thermal hysteresis of the prototypal spin-transition $\mathrm{Fe}$ II(phen)(2)(NCS)(2) compound via the microcrystallites environment: Experiments and mechanoelastic model, J. Mater. Chem. 22, 20451 (2012).

[36] Y. Chen, J.-G. Ma, J.-J. Zhang, W. Shi, P. Cheng, D.-Z. Liao, and S.-P. Yan, Spin crossover-macromolecule composite nano film material, Chem. Commun. 46, 5073 (2010).

[37] S.-W. Lee, J.-W. Lee, S.-H. Jeong, I.-W. Park, Y.-M. Kim, and J.-I. Jin, Processable magnetic plastics composites-spin crossover of PMMA/Fe(II)-complexes composites, Synth. Met. 142, 243 (2004).

[38] V. Martinez, I. Boldog, A. B. Gaspar, V. Ksenofontov, A. Bhattacharjee, P. Gütlich, and J. A. Real, Spin crossover phenomenon in nanocrystals and nanoparticles of $\left[\mathrm{Fe}(3-\mathrm{Fpy})_{2} \mathrm{M}(\mathrm{CN})_{4}\right]$ $\left(\mathrm{M}^{\mathrm{II}}=\mathrm{Ni}, \mathrm{Pd}, \mathrm{Pt}\right)$ two-dimensional coordination polymers, Chem. Mater. 22, 4271 (2010).

[39] M. Rubio, R. Hernández, A. Nogales, A. Roig, and D. López, Structure of a spin-crossover Fe(II) - 1,2,4-triazole polymer complex dispersed in an isotactic polystyrene matrix, Eur. Polym. J. 47, 52 (2011).

[40] J. Larionova, L. Salmon, Y. Guari, A. Tokarev, K. Molvinger, G. Molnar, and A. Bousseksou, Towards the ultimate size limit of the memory effect in spin-crossover solids, Angew. Chem. Int. Ed. 47, 8236 (2008).

[41] P. Durand, S. Pillet, E. E. Bendeif, C. Carteret, M. Bouazaoui, H. El Hamzaoui, B. Capoen, L. Salmon, S. Hebert, J. Ghanbaja, L. Aranda, and D. Schaniel, Room temperature bistability with wide thermal hysteresis in a spincrossover silica nanocomposite, J. Mater. Chem. C 1, 1933 (2013).

[42] A. Tissot, J.-F. Bardeau, E. Rivière, F. Brisset, and M.-L. Boillot, Thermo- and photoswitchable spin-crossover nanoparticles of an iron(II) complex trapped in transparent silica thin films, Dalton Trans. 39, 7806 (2010).

[43] S. Titos-Padilla, J. M. Herrera, X.-W. Chen, J. J. Delgado, and E. Colacio, Bifunctional hybrid $\mathrm{SiO}_{2}$ nanoparticles showing synergy between core spin crossover and shell luminescence properties, Angew. Chem. Int. Ed. Engl. 50, 3290 (2011).

[44] C. Faulmann, J. Chahine, I. Malfant, D. de Caro, B. Cormary, and L. Valade, A facile route for the preparation of nanoparticles of the spin-crossover complex $\left[\mathrm{Fe}(\mathrm{Htrz})_{2}(\operatorname{trz})\right]\left(\mathrm{BF}_{4}\right)$ in xerogel transparent composite films, Dalton Trans. 40, 2480 (2011).

[45] A. Bousseksou, J. Nasser, J. Linares, K. Boukheddaden, and F. Varret, Ising-like model for the two-step spin-crossover, J. Phys. I 2, 1381 (1992).

[46] T. Kawamoto and S. Abe, Thermal hysteresis loop of the spinstate in nanoparticles of transition metal complexes: Monte Carlo simulations on an Ising-like model, Chem. Commun. 0, 3933 (2005).

[47] T. Kawamoto and S. Abe, Monte Carlo simulations of an Isinglike model for photoinduced spin-state switching in nanoparticles of transition metal complexes, J. Phys.: Conf. Ser. 21, 56 (2005).

[48] A. Muraoka, K. Boukheddaden, J. Linarès, F. Varret, J. Linares, and F. Varret, Two-dimensional Ising-like model with specific edge effects for spin-crossover nanoparticles: A Monte Carlo study, Phys. Rev. B 84, 054119 (2011).

[49] D. Chiruta, C.-M. Jureschi, J. Linares, Y. Garcia, and A. Rotaru, Lattice architecture effect on the cooperativity of spin transition coordination polymers, J. Appl. Phys. 115, 053523 (2014).

[50] A. Atitoaie, R. Tanasa, A. Stancu, and C. Enachescu, Study of spin crossover nanoparticles thermal hysteresis using FORC diagrams on an Ising-like model, J. Magn. Magn. Mater. 368, 12 (2014).

[51] J. A. Nasser, First order high-spin/low-spin phase transition induced by acoustic-phonons, Eur. Phys. J. B 21, 3 (2001).

[52] J. A. Nasser, K. Boukheddaden, and J. Linares, Two-step spin conversion and other effects in the atom-phonon coupling model, Eur. Phys. J. B 39, 219 (2004).

[53] K. Boukheddaden, S. Miyashita, and M. Nishino, Elastic interaction among transition metals in one-dimensional spin-crossover solids, Phys. Rev. B 75, 094112 (2007). 
[54] K. Boukheddaden, M. Nishino, and S. Miyashita, Molecular Dynamics and Transfer Integral Investigations of an Elastic Anharmonic Model for Phonon-Induced Spin Crossover, Phys. Rev. Lett. 100, 177206 (2008).

[55] M. Nishino, K. Boukheddaden, Y. Konishi, and S. Miyashita, Simple Two-Dimensional Model for the Elastic Origin of Cooperativity Among Spin States of Spin-Crossover Complexes, Phys. Rev. Lett. 98, 247203 (2007).

[56] C. Enachescu, L. Stoleriu, A. Stancu, and A. Hauser, Model for Elastic Relaxation Phenomena in Finite 2D Hexagonal Molecular Lattices, Phys. Rev. Lett. 102, 257204 (2009).

[57] W. Nicolazzi, S. Pillet, and C. Lecomte, Two-variable anharmonic model for spin-crossover solids: A like-spin domains interpretation, Phys. Rev. B 78, 174401 (2008).

[58] H. Oubouchou, A. Slimani, and K. Boukheddaden, Interplay between elastic interactions in a core-shell model for spincrossover nanoparticles, Phys. Rev. B 87, 104104 (2013).

[59] M. Mikolasek, W. Nicolazzi, F. Terki, G. Molnár, and A. Bousseksou, Surface transition in spin crossover nanoparticles, Chem. Phys. Lett. 678, 107 (2017).

[60] M. Mikolasek, W. Nicolazzi, F. Terki, G. Molnár, and A. Bousseksou, Investigation of surface energies in spin crossover nanomaterials: The role of surface relaxations, Phys. Chem. Chem. Phys. 19, 12276 (2017).

[61] C. Enachescu, R. Tanasa, A. Stancu, A. Tissot, J. Laisney, and M.-L. Boillot, Matrix-assisted relaxation in $\mathrm{Fe}(\mathrm{phen})_{2}(\mathrm{NCS})_{2}$ spin-crossover microparticles, experimental and theoretical investigations, Appl. Phys. Lett. 109, 031908 (2016).

[62] A. Slimani, H. Khemakhem, and K. Boukheddaden, Structural synergy in a core-shell spin crossover nanoparticle investigated by an electroelastic model, Phys. Rev. B 95, 174104 (2017).

[63] L. Stoleriu, A. Stancu, P. Chakraborty, A. Hauser, and C. Enachescu, Analysis of first order reversal curves in the thermal hysteresis of spin-crossover nanoparticles within the mechanoelastic model, J. Appl. Phys. 117, 17B307 (2015).

[64] F. Volatron, L. Catala, E. Riviere, A. Gloter, O. Stephan, and T. Mallah, Spin-crossover coordination nanoparticles, Inorg. Chem. 47, 6584 (2008).

[65] A. Slimani, K. Boukheddaden, and K. Yamashita, Thermal spin transition of circularly shaped nanoparticles in a core-shell structure investigated with an electroelastic model, Phys. Rev. B 89, 214109 (2014).

[66] A. Slimani, K. Boukheddaden, F. Varret, H. Oubouchou, M. Nishino, and S. Miyashita, Microscopic spin-distortion model for switchable molecular solids: Spatiotemporal study of the deformation field and local stress at the thermal spin transition, Phys. Rev. B 87, 014111 (2013).

[67] A. Slimani, K. Boukheddaden, F. Varret, M. Nishino, and S. Miyashita, Properties of the low-spin high-spin interface during the relaxation of spin-crossover materials, investigated through an electro-elastic model, J. Chem. Phys. 139, 194706 (2013).

[68] A. Slimani, K. Boukheddaden, and K. Yamashita, Effect of intermolecular interactions on the nucleation, growth, and propagation of like-spin domains in spin-crossover materials, Phys. Rev. B 92, 014111 (2015).

[69] G. Félix, W. Nicolazzi, M. Mikolasek, G. Molnár, and A. Bousseksou, Non-extensivity of thermodynamics at the nanoscale in molecular spin crossover materials: A balance between surface and volume, Phys. Chem. Chem. Phys. 16, 7358 (2014).
[70] K. Boukheddaden, A. Slimani, M. Sy, F. Varret, O. Hassane, and T. Rachid, Physical Properties of 2D Spin-Crossover Solids from an Electro-Elastic Description: Effect of Shape, Size, and Spin-Distortion Interactions (Pan Stanford, New York, 2016).

[71] G. Félix, M. Mikolasek, G. Molnár, W. Nicolazzi, and A. Bousseksou, Tuning the spin crossover in nano-objects: From hollow to core-shell particles, Chem. Phys. Lett. 607, 10 (2014).

[72] M. Mikolasek, G. Félix, G. Molnár, F. Terki, W. Nicolazzi, and A. Bousseksou, Role of surface vibrational properties on cooperative phenomena in spin-crossover nanomaterials, Phys. Rev. B 90, 075402 (2014).

[73] G. Félix, M. Mikolasek, G. Molnár, W. Nicolazzi, and A. Bousseksou, Control of the phase stability in spin-crossover core-shell nanoparticles through the elastic interface energy, Eur. J. Inorg. Chem. 2018, 435 (2018).

[74] K. Rodpun, Synthesis of Cu-nanocrystal coordination polymer by microemulsion method. Straightforward experiment of nanomaterial synthesis for science high school students, World J. Chem. Educ. 5, 107 (2017).

[75] A. Slimani, F. Varret, K. Boukheddaden, D. Garrot, H. Oubouchou, and S. Kaizaki, Velocity of the High-Spin Low-Spin Interface Inside the Thermal Hysteresis Loop of a Spin-Crossover Crystal, via Photothermal Control of the Interface Motion, Phys. Rev. Lett. 110, 087208 (2013).

[76] W. Wernsdorfer, E. Bonet Orozco, K. Hasselbach, A. Benoit, D. Mailly, O. Kubo, H. Nakano, and B. Barbara, Macroscopic Quantum Tunneling of Magnetization of Single Ferrimagnetic Nanoparticles of Barium Ferrite, Phys. Rev. Lett. 79, 4014 (1997).

[77] E. Bonet, W. Wernsdorfer, B. Barbara, A. Benoît, D. Mailly, and A. Thiaville, Three-Dimensional Magnetization Reversal Measurements in Nanoparticles, Phys. Rev. Lett. 83, 4188 (1999).

[78] A. Tokarev, J. Long, Y. Guari, J. Larionova, F. Quignard, P. Agulhon, M. Robitzer, G. Molnár, L. Salmon, and A. Bousseksou, Spin crossover polysaccharide nanocomposites, New J. Chem. 37, 3420 (2013).

[79] D. Qiu, D.-H. Ren, L. Gu, X.-L. Sun, T.-T. Qu, Z.-G. Gu, and Z. $\mathrm{Li}$, Spin crossover-graphene nanocomposites: Facile syntheses, characterization, and magnetic properties, RSC Adv. 4, 31323 (2014).

[80] C. Gatel, F. J. Bonilla, A. Meffre, E. Snoeck, B. Warot-Fonrose, B. Chaudret, L.-M. Lacroix, and T. Blon, Size-specific spin configurations in single iron nanomagnet: From flower to exotic vortices, Nano Lett. 15, 6952 (2015).

[81] L.-M. Lacroix, F. N. Huls, D. Ho, X. Sun, K. Cheng, and S. Sun, Stable single-crystalline body centered cubic Fe nanoparticles, Nano Lett. 11, 1641 (2011).

[82] A. Slimani, F. Varret, K. Boukheddaden, C. Chong, H. Mishra, J. Haasnoot, and S. Pillet, Visualization and quantitative analysis of spatiotemporal behavior in a first-order thermal spin transition: A stress-driven multiscale process, Phys. Rev. B 84, 094442 (2011).

[83] M. Sy, F. Varret, K. Boukheddaden, G. Bouchez, J. Marrot, S. Kawata, and S. Kaizaki, Structure-driven orientation of the high-spin-low-spin interface in a spin-crossover single crystal, Angew Chem. Int. Ed. 53, 7539 (2014).

[84] H. Fourati, E. Milin, A. Slimani, G. Chastanet, Y. Abid, S. Triki, and K. Boukheddaden, Interplay between a crystal's 
shape and spatiotemporal dynamics in a spin transition material, Phys. Chem. Chem. Phys. 20, 10142 (2018).

[85] E. Milin, V. Patinec, S. Triki, E.-E. Bendeif, S. Pillet, M. Marchivie, G. Chastanet, and K. Boukheddaden, Elastic frustration triggering photoinduced hidden hysteresis and multistability in a two-dimensional photoswitchable Hofmann-like spincrossover metal-organic framework, Inorg. Chem. 55, 11652 (2016).

[86] N. Ortega-Villar, M. Muñoz, and J. Real, Symmetry breaking in iron(II) spin-crossover molecular crystals, Magnetochemistry 2 , 16 (2016).

[87] A. J. Fitzpatrick, E. Trzop, H. Müller-Bunz, M. M. Dîrtu, Y. Garcia, E. Collet, and G. G. Morgan, Electronic vs. structural ordering in a manganese(III) spin crossover complex, Chem. Commun. 51, 17540 (2015).

[88] M. Paez-Espejo, M. Sy, and K. Boukheddaden, Elastic frustration causing two-step and multistep transitions in spin-crossover solids: Emergence of complex antiferroelastic structures, J. Am. Chem. Soc. 138, 3202 (2016).

[89] R. Traiche, M. Sy, and K. Boukheddaden, Elastic frustration in 1D spin-crossover chains: Evidence of multi-step transitions and self-organizations of the spin states, J. Phys. Chem. C 122, 4083 (2018).

[90] J. Jung, F. Bruchhäuser, R. Feile, H. Spiering, and P. Gütlich, The cooperative spin transition in $\left[\mathrm{Fe}_{x} \mathrm{Zn}_{1-x}(\mathrm{ptz})_{6}\right]\left(\mathrm{BF}_{4}\right)_{2}$ : I. Elastic properties-An oriented sample rotation study by Brillouin spectroscopy, Z. Phys. B: Condens. Matter 100, 517 (1996).
[91] H. Spiering, K. Boukheddaden, J. Linares, and F. Varret, Total free energy of a spin-crossover molecular system, Phys. Rev. B 70, 184106 (2004).

[92] See Supplemental Material at http://link.aps.org/supplemental/ 10.1103/PhysRevB.98.014106 for Fig. S1: Spatial map of the distribution of the local pressure over the lattice along the thermal hysteresis loop and Fig. S2: Thermal variation of the elastic energy density of (a) shell and (b) core, along the heating branch of the thermal hysteresis, for different shell thicknesses.

[93] M. Nishino, C. Enachescu, S. Miyashita, P. A. Rikvold, K. Boukheddaden, and F. Varret, Macroscopic nucleation phenomena in continuum media with long-range interactions, Sci. Rep. 1, 162 (2011).

[94] C. Enachescu, M. Nishino, S. Miyashita, K. Boukheddaden, F. Varret, and P. A. Rikvold, Shape effects on the cluster spreading process of spin-crossover compounds analyzed within an elastic model with Eden and Kawasaki dynamics, Phys. Rev. B 91, 104102 (2015).

[95] C. Enachescu, M. Nishino, S. Miyashita, L. Stoleriu, and A. Stancu, Monte Carlo Metropolis study of cluster evolution in spin-crossover solids within the framework of a mechanoelastic model, Phys. Rev. B 86, 054114 (2012).

[96] L. Stoleriu, M. Nishino, S. Miyashita, A. Stancu, A. Hauser, and C. Enachescu, Cluster evolution in molecular three-dimensional spin-crossover systems, Phys. Rev. B 96, 064115 (2017).

[97] G. Rossi, R. Ferrando, and C. Mottet, Structure and chemical ordering in CoPt nanoalloys, Faraday Discuss. 138, 193 (2008). 